\title{
Rotor angle transient stability methodologies of power systems: a comparison
}

\begin{abstract}
Today's power system grids are massively interconnected with newer technologies and control devices integrated into the system all for enhanced efficiency and economic benefits in operation. This property makes the grid highly dynamic and diverse thus, concluding it as the biggest artificial living network on earth. To observe the power system security requirements, planning and safe operation of today's system becomes imperatively necessary. If the power system security criterion is ignored, then power blackouts will be born and the system response will distribute severe socio-economic impact, therefore, it is essential to know the dynamic security assessment (DSA) for the systems and allow it to remain in a stable state. This paper will execute a comparison of each method of transient stability index (TSI) and transient stability assessment (TSA) and how the classes of these methods are implemented. The classifications will highlight the difference between the methods and show the individual benefits and setbacks when applied on a practical large sized power system. This review paper can deliver an in-depth knowledge into the development of a rapid and reliable rotor angle transient instabilities technique towards improving DSA of a practical large-sized power system.
\end{abstract}

Keyword: Power system; Rotor angle transient stability; Transient stability index; Transient stability assessment 